\section{Submerged electrodes in an aquarium: Validation of a technique for remote sensing of behavior*}

\author{
DAVID A. GOODMAN $†$ and NORMAN M. WEINBERGER \\ Department of Psychobiology \\ University of California, Irvine, Irvine, California 92664
}

Necturus maculosus has been selected as representative of a class of neotenic amphibia whose behavior (including gill beat, heartbeat, and tail waving) can be sensed remotely by submerged electrodes in an aquarium. This communication presents a validation of the technique as a preface to reports on the electrophysiology of behavior in unrestrained surgically naive salamanders. Although the sources of the submarine signals are not known with certainty, they apparently depend on two factors: (1) aquarium water of appropriate ionic strength serves as a volume conductor for activity, and (2) animal movement displaces water or produces waves which are sensed as a change in potential at the water-electrode interface. This method provides a continuous comprehensive record of behavior simultaneously from several response systems in a freely behaving animal.

The purpose of this communication is to describe and present validation for a method of recording both behavioral and physiological data from unrestrained aquatic amphibia. This tech nique consists of implanting electrodes in the walls and floor of an aquarium rather than in the animal itself. Hereafter, we refer to this general method by the acronym SEA, "submerged electrodes in an aquarium." 1 We have used SEA systematically to investigate the behavior patterns of the wholly aquatic salamander, Necturus maculosus (Goodman, 1969). An understanding of these findings, which will be the subject of further communications, depends upon a prior understanding of SEA. This paper presents a validation of SEA as used for remote sensing of gill beat, heartbeat, and tail movements.

\section{MATERIALS AND METHODS Subjects}

Necturus maculosi, more commonly known as mud puppies, were obtained from the Lemberger Company, Oshkosh, Wisconsin, and housed in aerated 20 -gal aquaria cooled to $19^{\circ} \mathrm{C}$. $S$ snout-vent length averaged $18 \mathrm{~cm}$ and whole length, $30 \mathrm{~cm}$.

Necturus is a wholly aquatic neotenic salamander. Its primary mode of respiration at $19^{\circ} \mathrm{C}$ consists of uptake of oxygen from water by

*Research supported by Grant MH 11250 from the National Institute of Health to N.M.W.

+Now at Newport Neuroscience Center, Box 4045, Irvine, California 92664 "rowing" its large external gills back and forth (Gans, 1970). These contain rich capillary beds through which gaseous exchange is achieved. This mode of respiration is of more than cursory interest, because it permits unequivocal observation of respiration and the rowing movements of the gills can be sensed at a distance by electrodes embedded in the aquarium walls and floor.

Necturus swims quite well and it also walks on the bottom, spending most of the time standing on the bottom of a stream, pond, or aquarium (Harris, 1959). Its major swimming movements are accomplished by lateral movements of the tail which comprises approximately $40 \%$ of the body length. The tail of the mud puppy often waves from side to side while the animal rests on the bottom, beating its gills and remaining otherwise motionless. This tail movement causes water turbulence, the effects of which can also be sensed by aquarium electrodes, as described below.

\section{Apparatus}

Remote recording of physiological and behavioral phenomena in Necturus is performed in a Plexiglas aquarium, $28 \times 12 \times 10 \mathrm{~cm}$. The salamander is free to move within the aquarium; also, it can scale the walls and escape into a $30 \times 30 \times 10 \mathrm{~cm}$ water jacket in which the aquarium rests. ${ }^{2}$ Both jacket and aquarium are housed in a refrigerator maintained at $19^{\circ} \mathrm{C}$ (Fig. 1). Two types of exteroceptive stimulation can be presented-shadow and vibration. A moving shadow is produced by sequential interruption of current to a parallel array of luminescent strips located $30.4 \mathrm{~cm}$ above the aquarium (Weinberger \& Goodman, 1969). (This type of device eliminates problems of possible changes in heart flux and vibration accompanying the shadow.) Vibration can be induced by a 6 -in. speaker located beneath the aquarium, which is driven by an oscillator at $60 \mathrm{~Hz}$. Stimulation is controlled by solid-state logic housed outside the chamber.

Electrodes embedded in the Plexiglas aquarium consist of stainless steel studs (Nu-way "male"), $35 \mathrm{~mm}$ in diam, which protrude $2 \mathrm{~mm}$ into the interior of the aquarium. Seventeen electrodes are used, 14 in the walls and 3 in the floor. These are placed symmetrically (Fig. 2). Wall electrodes are located approximately $1 \mathrm{~cm}$ below the surface of the water. Electrodes are soldered to low-capacitance cables, which are insulated and waterproofed where they penetrate the outside of the aquarium, and led to a standard polygraph electrode board and then to a rotary selector switch panel which permits rapid selection of desired electrodes.

Recording is achieved with a standard polygraph (Grass Model 7), using high-gain ac-coupled differential preamplifiers (7 P 5). Various time

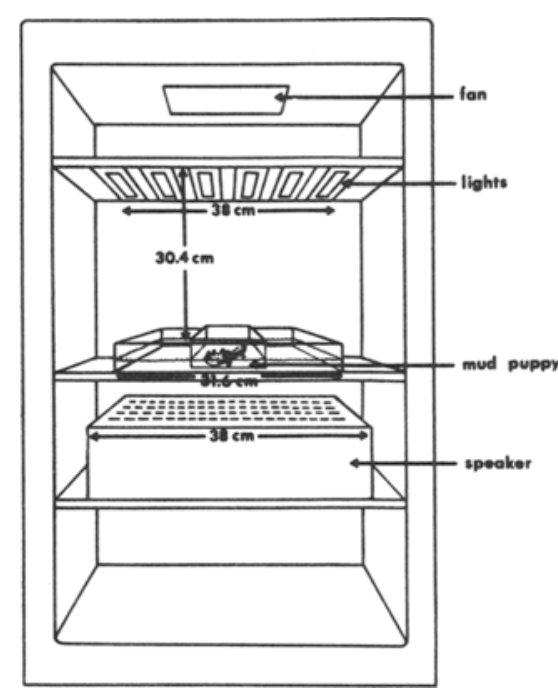

Fig. 1. Testing chamber with mud puppy in testing aquarium. The shadow apparatus is overhead ("lights") and the vibrational apparatus ("speaker") is below the aquarium. 


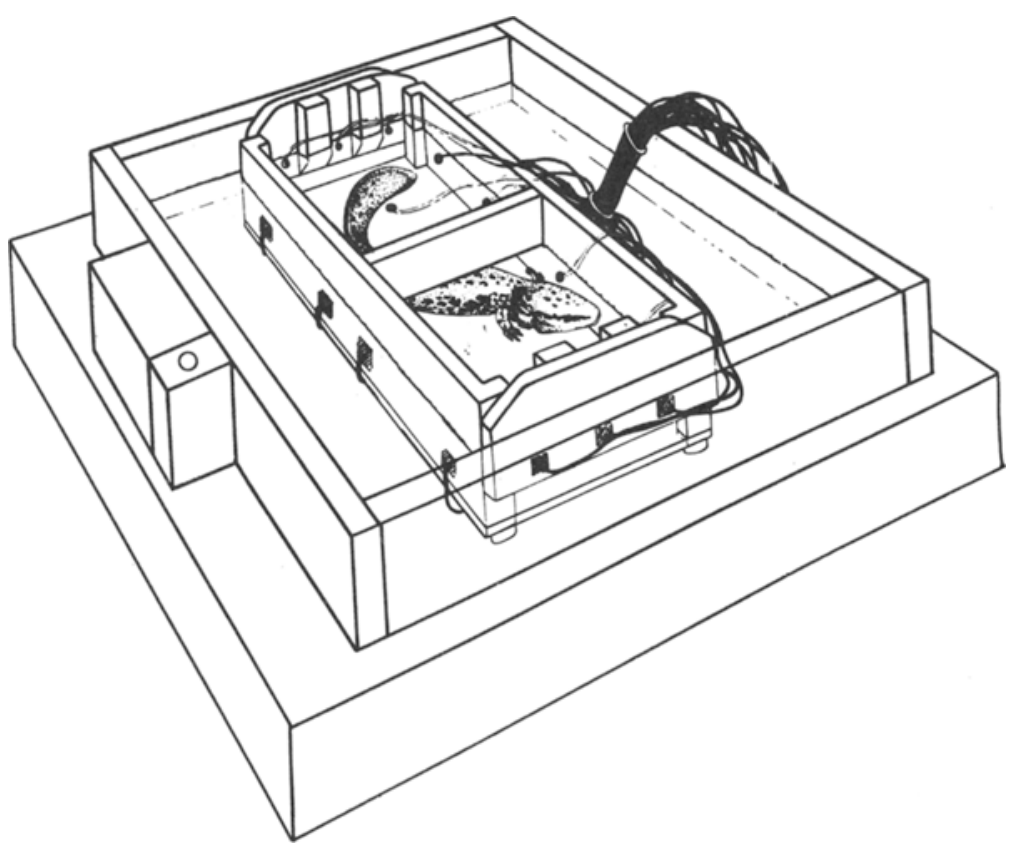

Fig. 2. Close-up of mud puppy in testing aquarium surrounded by the water jacket. Note the placement of electrodes in the walls and floor of the testing aquarium.

constants are used, as described in the figure legends.

\section{RESULTS}

When a salamander is quiescent, standing on the bottom, "rowing" its gills and rhythmically moving its tail, the selection of electrode pairs across the head, trunk, and tail reveals voltages which seem to be products of gill beat (respiration), heartbeat (EKG), and tail waving, respectively (Fig. 3). Both casual and invited observers have agreed that these potentials are isomorphic with the biological events with which they appear to coincide. However, such consensus provides insufficient evidence that the SEA technique does index these organismic processes. Accordingly, we have sought more objective validation of the technique. Two methods have been used to achieve this end: comparison of SEA potentials with (1) direct visual observation of gill and tail movement and (2) electrical potentials recorded by direct implantation of electrodes (flexible wires insulated except at the tips) in the gill stalks and in the heart.

\section{Gill Beat}

The gill beats of Necturus are characterized by rapid aft strokes followed by somewhat slower return strokes forward. Beating of the left and right gills in the quiescent salamander is generally synchronous. A comparison of gill beating observed directly with that recorded by
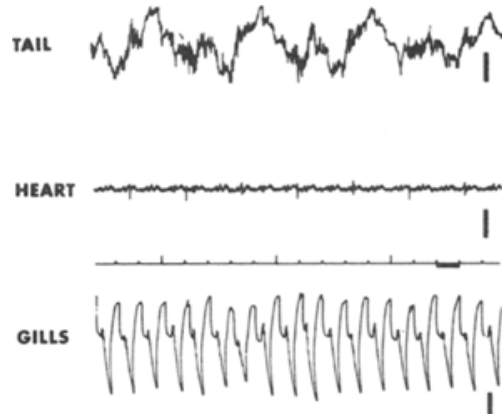

Fig. 3. Records of tail waving (TAIL), heartbeat (HEART) and gill beat (GILL) from electrode pairs across tail, thorax, and head, respectively. Calibrations: 1 sec and 10 microV. Bandpass in this and subsequent figures: tail, $0.1-75 \mathrm{~Hz}$; heart, 10-75 Hz; gills, 3-35 Hz.

did not interfere with gill beating. A typical record of gill-beat activity so recorded consists of a rapid potential corresponding to the rapid aft stroke and a slower potential of opposite polarity accompanying the forward return stroke (Fig. 5A). The SEA electrodes, located on either side of the head, simultaneously recorded potentials isomorphic with the gill waves. They differ from the directly recorded gill beats in an important way: they are broader and often lag behind the directly recorded waves by 50 to a maximum of $200 \mathrm{msec}$. This difference, together with the isomorphism of the direct and remotely recorded potentials, is to be expected if the SEA potentials are caused by water movement consequent to gill beating. The possible physical events responsible for the potentials recorded by SEA will be discussed in a later section. depressor muscles of one gill stalk. This electrode was quite flexible and
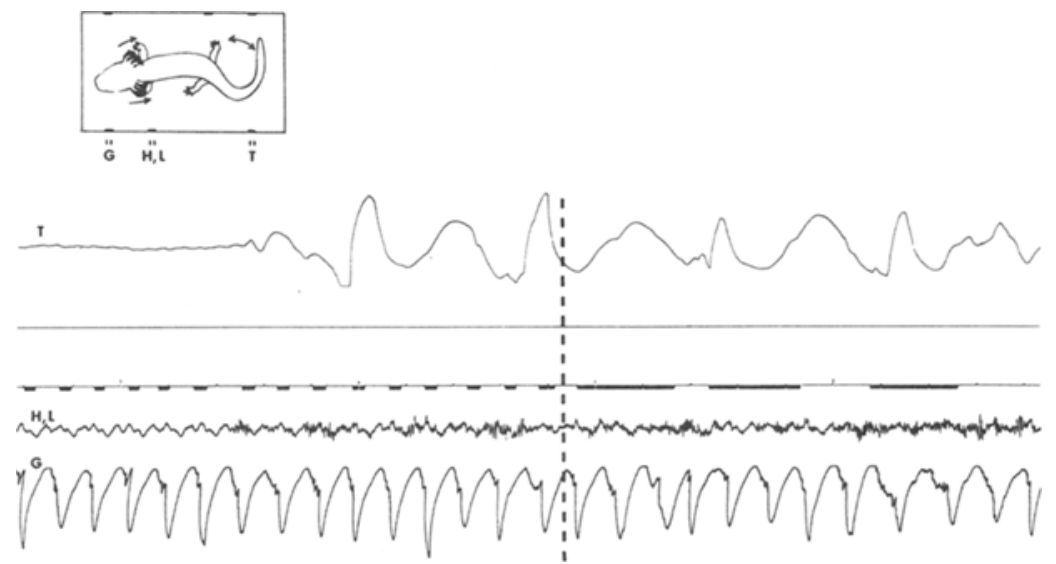

Fig. 4. SEA recording of tail (T) and gill (G) movement compared to direct visual observation. To the left of the vertical dotted line, the $E$ depressed the signal marker once per gill beat; to the right of the line, he did so once per tail wave. Note the correspondence between SEA and observed records. Electrodes near the trunk $(H, L)$ recorded fast waves, suggestive of EMG, during tail waving only. 


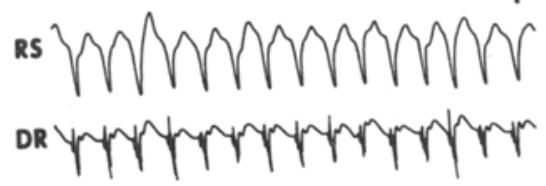

B

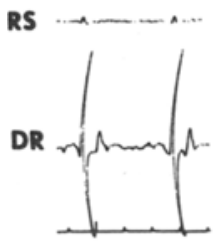

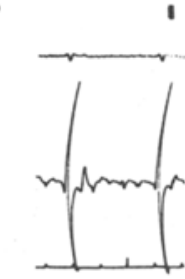

Fig. 5. Comparison of remotely sensed (RS) and directly recorded (DR) gill beat (A) and heartbeat (B). In $A$, there is perfect correspondence between $R S$ and DR. B illustrates inversion of remotely sensed heartbeat when mud puppy is rotated $180 \mathrm{deg}$-from facing north (n) to facing south (s). Remotely sensed heartbeat signal corresponds to QRS complex of EKG. Calibrations: $1 \mathrm{sec}$ and 10 microV.

Interruption of gill beating comprises one important component of the mud puppy orienting reflex (Goodman \& Weinberger, 1970). Figure 6 illustrates the effect of an overhead moving shadow upon gill beating recorded both directly and remotely. Note the correspondence between the two records, with the remote recording lagging slightly behind the directly recorded potentials, including slightly longer latency to gill-beat interruption and restart after a quiescent period of about 17 sec. These findings offer support for the notion that the SEA potentials can serve as an adequate index of gill beating in the quiescent salamander. However, it must be noted that the latency measures calculated from SEA potentials must take into account the time lag between the actual gill beat and that representation recorded by the remote electrodes. This lag is a function of the distance between the gills and electrodes, which for most of our data is on the order of $1.0-2.0 \mathrm{~cm}$. Relative latencies, obtained from the same animal which has not moved its head between trials, are unaffected.

\section{Heartbeat}

Presumptive heartbeat potentials recorded by SEA are quite small, often on the order of 5 to 10 microV. A comparison of such potentials with the EKG recorded directly with a wire electrode inserted into the surface of

Fig. 6. Same animal as in $5 \mathrm{~A}$, presented with a sudden shadow (S). Cessation of gill beat is noted with directly recorded (DR) and remotely sensed (RS) record. Shadow intensity indicated in $\mathrm{S}$ measured by photocell to left of testing aquarium (Fig. 2).

the ventricle is presented in Fig. $5 \mathrm{~B}$. The SEA potentials were recorded from electrodes on either side of the trunk. If, in fact, the remote recording was of the moving dipole which is associated with the heartbeat and depolarization-repolarization of the myocardium, it would be expected that as the animal was rotated $180 \mathrm{deg}$ its remotely sensed heartbeat signal would show a polarity inversion. The direct EKG should not be altered. These expectations were confirmed (Fig. 5B).

Unlike the SEA gill-beat potentials, which seem to be due to wave propagation to the recording electrodes, the remotely sensed heartbeat does not lag behind that recorded directly. This suggests that the latter phenomenon is not a function of wave propagation, but is, rather, a recording of a volume-conducted potential at a distance. Additional evidence favoring this interpretation is shown in Fig. 7 which indicates that the SEA-sensed heartbeat amplitude is smaller than that recorded directly, and also that SEA electrodes must be in the immediate vicinity of the animal in order to register its heartbeat. This figure also indicates that the noise level of recordings increases with greater distance between selected electrodes. This effect may be a function of the associated increasing resistance between electrodes

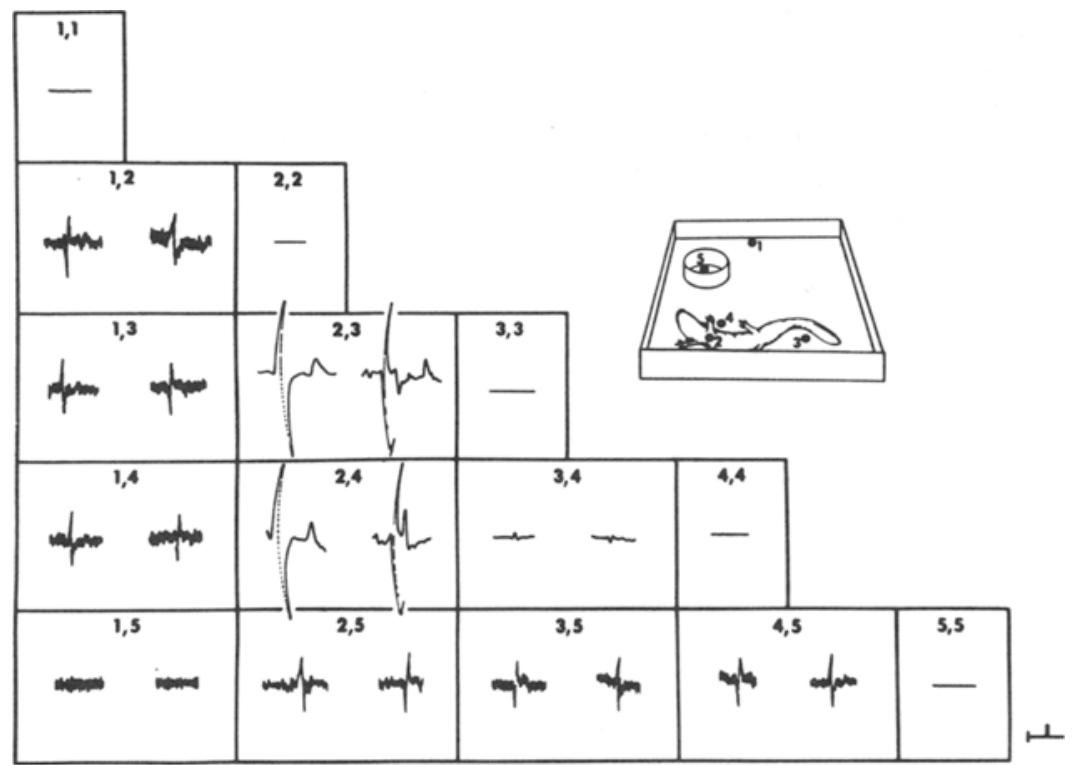

Fig. 7. The heart signal recorded both directly and remotely. Electrode 2 was in the myocardium. Electrodes 3 and 4 were in the water on either side of the trunk, approximately equidistant from the heart. No. 1 was also in the water, distal to the animal, and No. 5 was in a beaker of water, insulated from the aquarium water. Shorted pairs of leads (e.g., "1,1") reveal an insignificant amount of amplifier noise. Note that all pairs except " 1,5 ," pick up the heart signal. Baseline noise increases with distance between selected electrodes. Pairs of records show recordings with both normal and reversed polarity. Calibrations: 1 sec and 10 microv. 

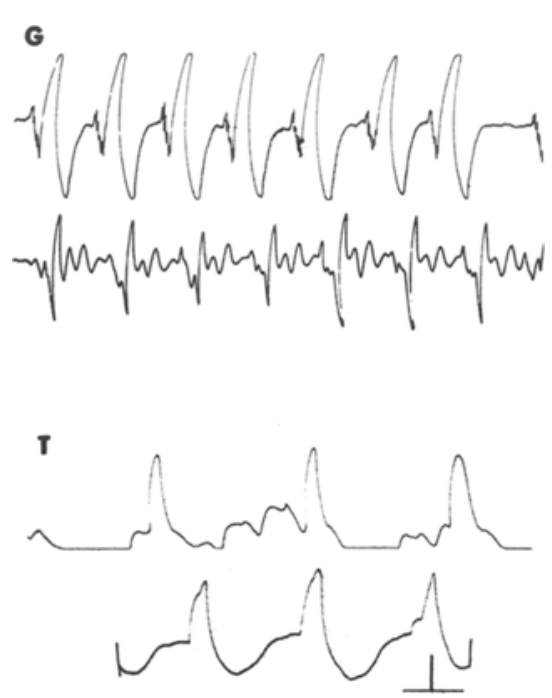

Fig. 8. An attempt at simulation of gill beat (G) and tail wave (T) signals, with a wooden paddle. Lower traces were simulated; upper traces were selected from real SEA records of mud puppy behavior. Calibrations: 1 sec and 10 microV.

separated by greater volumes of water. If two electrodes are chosen such that they are both quite close to the salamander, the background noise and heart signal will both be quite small. This result is also consonant with volume conduction theory in that two electrodes near the animal will lie near identical isopotential contours; the use of differential amplifiers causes great attenuation of common-mode signals, and hence the small amplitude of the resultant waveform. This is not necessarily a handicap, however, for the common-mode noise is attenuated to at least an equal degree (see Fig. 7 ,

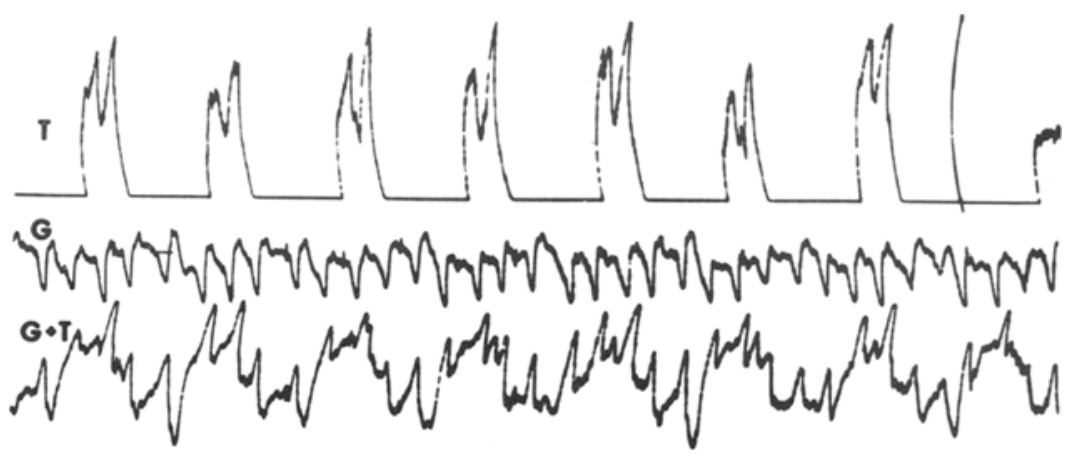

Fig. 9. Tail wave record (T) as sensed by a pair of caudal electrodes; gill-beat record (G) sensed by pair of anterior electrodes. $G+T$ record is from an electrode pair midway between tail and head. The tail record is "clipped" because the tail was actually hitting an electrode. Calibrations: 1 sec.

" 3,4 "). As long as there is an adequate signal-noise ratio, an unmistakable heartbeat signal can be extracted by electronic means such as bandpass filtering (Goodman \& Weinberger, in press).

\section{Tail Movement}

In the quiescent state, Necturus often waves its tail rhythmically from side to side, at a frequency of 0.2-0.3 Hz. Figure 4 (right side) depicts the one-to-one correspondence between the SEA-recorded tail potentials (T) and those observed by the $E$ and indicated by the stimulus marker, without reference to the polygraph write-out. Tail activity is easily distinguished from gill activity because gill beating is at a higher frequency $\left(0.9-1.3 \mathrm{~Hz}\right.$ at $\left.19^{\circ} \mathrm{C}\right)$. Additionally, tail waving is evident at caudal electrodes, while gill beat is recorded from rostral electrodes (Fig. 3).

Both the tail wave record and the gill-beat record probably derive from similar sources. If the gill or tail movement takes place close to the electrode, both a myographic (BMG) component (volume conducted) and a slow potential fluctuation are present. A small myographic component in the tail record is suggested by Fig. 3 (tail), Fig. 5 ("H,L"), and from direct recording in an earlier study (Goodman \& Weinberger, 1969).

It seems likely that the mechanism of SEA tail potentials is similar to that for the gill-beat potentials, that is, that they are consequent to water movement caused by movement of the tail. There can be a distinct lag from the animal and those recorded from the remote electrodes. Additionally, the SEA waveform is broader, that is, spread over time (Fig. 6A). In an attempt to produce synthetic gill or tail potentials, we used a small wooden paddle to simulate gill and tail movements (Fig. 8). Potentials were produced primarily by the condensation wave. These findings support the view that the large SEA gill and tail potentials between the signals recorded directly

H

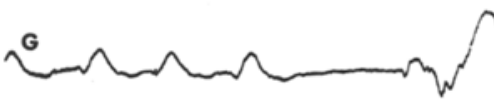

s

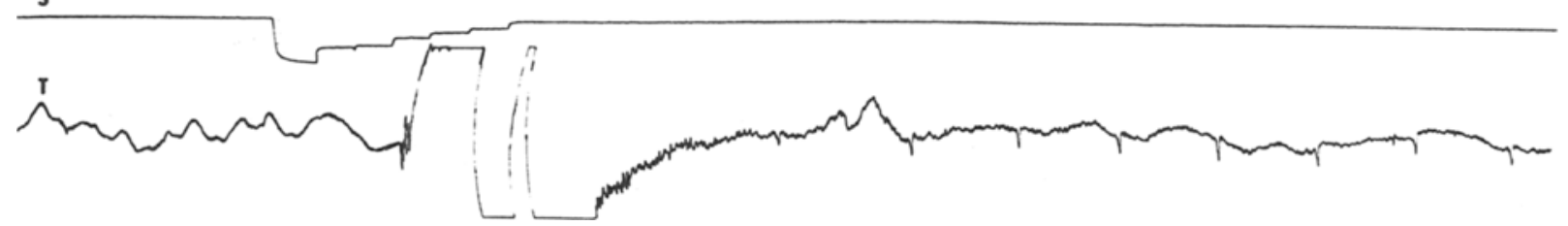

Fig. 10. SEA record of retreat from overhead shadow (S). Prior to the shadow, heartbeat (H) and gill beat (G) were regular; the tail (T) was not waving. Mud puppy response to stimulus was interruption of ongoing activity followed by rapid movement backwards. The heartbeat reappears at the tail ( $T$ ) electrodes indicating the mud puppy's thorax is now where its tail was. Calibrations: 1 sec. 


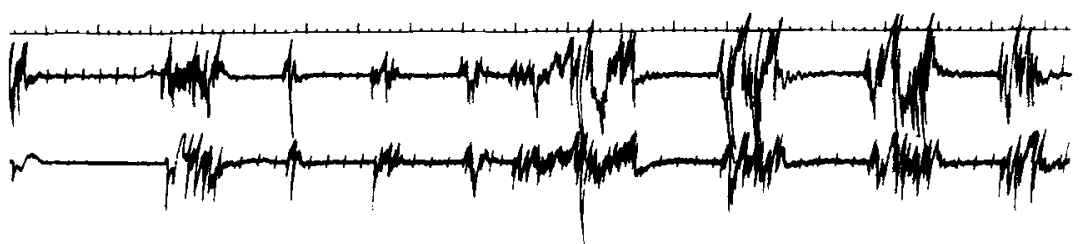

Fig. 11. Tracking a moving mud puppy as it moves forwards. Upper trace from electrode pair midway along aquarium walls; lower trace from anterior wall. Animal struggles rhythmically to escape through opening in anterior wall. Note the shift in heart signal to the anterior electrodes.

are due to waves reaching the recording electrodes. Finally, if tail-beat and gill-beat records are consequences of water movement, electrodes close to the tail should sense tail movement, not gill movement, while gill electrodes should record gill, not tail, movement; electrodes midway between gills and tail should sense both signals. These expectations were fulfilled (Fig. 9).

\section{Animal Movement}

The gill, heart, and tail signals are obscured during gross movements of the animal (Figs. 10 and 11). Thus, absence of a heart signal under such conditions should not be interpreted as absence of heart beating in the animal. However, such movement produces its own record in the form of large variable potentials. Thus a record of the animal's behavior is continually produced. It cannot move without causing voltage changes at recording electrodes, probably due to waves or water turbulence (Fig. 10). In practice, the animal can easily be relocated following subsidence of large movement. This can be done without viewing the animal, if desired, by systematic choice of electrode pairs throughout the aquarium to produce a satisfactory heart or gill signal. For example, mud puppies may back up in response to an overhead shadow. Figure 10 depicts one such instance. Note that the onset of shadow is followed by disappearance of the heart signal from Channel $H$ and its reappearance at the set of former tail electrodes ("T") located approximately $4 \mathrm{~cm}$ posterior to the heart electrodes. Visual observation confirmed the fact that the animal had retreated. Thus the animal can be tracked in the aquarium in the absence of direct visual observation by noting the shift in locus of the SEA heart signal as the animal shifts position (Fig. 11).

\section{DISCUSSION}

Advantages of SEA

We have presented evidence which indicates that gill beating, heartbeating, and tail waving can be recorded remotely in the unrestrained mud puppy. The implications of these findings include the possibility of objectively obtaining a written record of the behavior of a generalized vertebrate which would be distorted or unobtainable under other conditions. These are its major advantages: (1) visual observation cannot provide detailed information about heartbeat or EMG; (2) a permanent record of heart, gill, and tail data also permits detailed analysis of their interactions and the effects of transient environmental stimuli upon them (Goodman, 1969; Goodman \& Weinberger, in press); (3) an animal may live in the testing chamber and need not be disturbed to affix transducers or inspect the leads; (4) the animal is free to move, and indeed cannot move without simultaneously producing some SEA record of its behavior by producing water displacement or turbulence.

Remote sensing with submerged electrodes in an aquarium yields a more complete picture of behavior than an approach which focuses upon a single component of complex action patterns. Additionally, it is more suited than visual observation in providing information about behavioral changes which cannot be seen (e.g., bradycardia to a shadow) or which do not reach threshold for gross movements (e.g., somatic EMG).

\section{Limitations of SEA}

The SEA technique does have limitations which must be discussed explicitly. These derive from (1) inability to identify with certainty the exact source of all signals: (2) small amplitude of the heart signal; (3) obliteration of signals when the animal moves; (4) signals from the gills and tail may be mixed and difficult to analyze; and (5) factors affecting signal amplitude.

(1) We do not know with certainty the physical causes of the SEA potentials. We have already indicated reasons for believing that the heart signal is a representation of the EKG, recorded by volume conduction.4 Briefly, its amplitude decreases with distance from the heart, and it occurs simultaneously with the EKG recorded directly. It seems highly likely that recording of the heart signal by the SEA technique does not differ in principle from the method used to record the EKG from the body surface under normal circumstances, that is, by volume conduction and the placement of electrodes on differing isopotential contours. Movement of the gills, tail, and whole body produce surface waves, subsurface water movement, or both, which appear to be propagated to the electrodes. The detailed nature of the possible biophysical events resulting in SEA potentials consequent to these events has been described elsewhere (Goodman \& Weinberger, in press).

(2) The heart signal may be very small (e.g., less than 10 microV). This problem is more apparent than real because it is the signal-to-noise ratio, not the absolute signal amplitude, which is critical. ${ }^{5}$ In practice, standard low-level biological amplifiers, which have been routinely balanced for optimal common-mode rejection ratio, are adequate to the task. The inherent noise level of such amplifiers is generally less than $2 \mathrm{microV}$. Additionally, active filtering (in the present case high-pass filtering at $10 \mathrm{~Hz}$ ) can attenuate unwanted potentials, leaving the heart signal clearly discernible.

(3) The heart, gill, and tail signals may be obliterated during gross movement. However, the movement itself produces large SEA potentials so that the animal produces a record of this behavior, even in the absence of direct visual observation. In practice, salamanders, once acclimatized to a novel environment, are notoriously lethargic. Thus, in practice, it has been possible to routinely record every heart, gill, and tail signal continuously for at least $90 \mathrm{~min}$.

(4) The salamander should be linear for best recording. Whenever the salamander becomes coiled or S-shaped, records such as those presented in Fig. 3 are not easily achieved. Nonetheless, original judicious placement of electrodes in the aquarium and careful selection of those electrodes, assures clear-cut gill-beat, heartbeat, and tail "beat" signals regardless of salamander orientation.

(5) Interpretation of the amplitude of SEA signals may be difficult. The variables which could affect amplitude, in addition to a bona fide change of the generator, include the interelectrode distance, resistance of the conducting medium, and orientation of the signal generators (e.g., heart, tail) with respect to the electrodes. Only in cases in which these variables are constant or their effects precisely known should 
variations in signal amplitude be attributed to a change in generator output. Our own proclivity continues to be to disregard largely absolute amplitude and to rely upon the SEA technique to provide clear indications of the occurrence of events and their temporal structure, rather than their magnitude. ${ }^{6}$ This limitation does not reduce the power of the technique to quantify the intervals between events or the duration of cessation of an event, e.g., gill-beat stoppage.

\section{Other Studies}

Remote sensing of one organismic function at a time has been attempted previously. Each technique has definite advantages and limitations; fish respiration was noted by Kleerekoper and Sibakin (1957) using remote voltage sensing, while Barham, Huckabay, Gowdy, and Burn (1969) measured "microvolt signals (EMG) from fishes and the environment." Other techniques have been used for a heartbeat record (Cohen \& Chandler, 1969; Tremor \& Rogallo, 1970), and for activity (Heusner \& Enright, 1966). Closest in scope to the present study is work by Gulyaev, Zabotin, and Schipperbakh (1968), who recorded frog bioelectric potentials at a distance with special high-impedance probes. To our knowledge, no previous investigators have attempted remote sensing of multiple functions simultaneously in unrestrained animals.

\section{SUMMARY}

In summary, SEA recording technique has been validated for gill beat, heartbeat, and tail waving. Mention has been made of the possibility that somatic EMG as well as gill-beat and tail-"beat" EMG can be sensed remotely. Measurement of these outputs simultaneously encourages comprehensive analysis of physiology and behavior in that important transitional vertebrate the neotenic salamander, Necturus maculosus.

\section{REFERENCES}

BARHAM, E. G., HUCKABAY, W. B., GOWDY, R., \& BURN, B. Microvolt electric signals from fishes and the environment. Science, 1969, 164, 965-968.

COHEN, D., \& CHANDLER, L. Measurements and a simplified interpretation of magnetocardiograms from humans. Circulation, 1969, 39 . 395-402.

GANS, C. Strategy and sequence in the evolution of the external gas exchanges of ectothermal vertebrates. Forma et Functio, 1970, 3, 61-104.

GOODMAN, D. A. Some brain reflexes of Necturus maculosus, the mud puppy. Unpublished doctoral dissertation. University of California, Irvine, 1969.

GOODMAN, D. A., \& WEINBERGER, N $M$. An electroencephalographic study of Necturus maculosus (mud puppy). Physiological Zoology, 1969, 42. 398-410.

GOODMAN, D. A.. \& WEINBERGER, N. $M$. Possible relationships between orienting and diving reflexes. Nature. $1970,225,1153-1154$.

GOODMAN, D. A., \& WEINBERGER, N. $M$. Remote sensing of behavior in aquatic amphibia especially in Necturus maculosus, the mud puppy. Communications in Behavioral Biology, in press.

GULYAEV, P. I., ZABOTIN, V. I., SH IP PENRAKH, N. Y. The electroauragram of the frog's nerve, muscle, and heart and of the human heart and musculature. Doklady Biological Science, 1968, 180, 359-361.

HARRIS, J. P., JR. The natural history of Necturus. I. Habitats and habits. Field \& Laboratory, 1959, 27, 11-20.

HEUSNER, A. A., \& ENRIGHT, J. T. Long term activity recording in small aquatic animals. Science, 1966, 154, 532-533.

KLEEREKOPER, H., \& SIBAKIN, K. An investigation of the "electric" spike potentials produced by the sea lamprey (Petromyzon marinus) in the wate surrounding the head region. Journal of the Fish Research Board, Canada, 1957, 14, 145-151.

M A C C H E T ELL I, F, J., \& MONT ANARELLI, N., JR. A simple chronic cortical electrode for the monkey. Journal of Experimental Animal Behavior, 1965, 4, 636-640.

TREMOR, J. W., \& ROGALLO. V. L. A small animal ballistocardiograph: Description and illustration of its use. Physiology \& Behavior, 1970, 5, 247-251. WEINBERGER, N. M. \& GOODMAN, D.

A. A solid-state device for presenting moving shadows. Behavior Research Methods \& Instrumentation, 1969, 1 192-193.

\section{NOTES}

1. This technique has been deseribed briefly in a previous communication (Goodman \& Weinberger, in press).

2. This provides an operational measure of "stress." Animals that remained in the SEA aquarium were considered to be unstressed.

3. "Nu-way" snap electrodes have been used before (Macchetelli \& Montanarelli, 1965)

4. The heart signal amplitude also depends upon the ionic strength of the water. Recordings are poor in pure water, but quite good in water inhabited by a mud puppy for a few days.

5. Signal-to-noise ratio for the heartbeat signal was sufficient for interval analysis using a Schmitt trigger and Nuclear Chicago Model DRC 7600. Heartbeat interval distributions between $11^{\circ} \mathrm{C}$ and $23^{\circ} \mathrm{C}$ are presented in Goodman (1969).

6. Although we stress the occurrence of signals, rather than their particular electrica characteristics, the following is a resumé of our routine findings: heart signal at 10-75 $\mathrm{Hz}$, biphasic, 5-15 microV peak-peak, $50 \mathrm{msec}$ duration; gill signal at $3-35 \mathrm{~Hz}$ biphasic, $30-50$ microv peak-peak, $500 \mathrm{msec}$ duration; tail at $0.1-75 \mathrm{~Hz}$ biphasic, $30-70$ microv peak-peak, $2,700-3,000$ msec duration. 